\title{
Les métropoles devront-elles subir un contrôle anti-dopage ?
}

Les territoires français bénéficiant du statut administratif de métropoles affichent des résultats souvent meilleurs en termes d'emplois que ceux des autres strates urbaines. Mais cela est-il lié à leur propre attractivité ou aux avantages financiers et réglementaires accordés par l'État ? En d'autres termes, les performances des métropoles ne sont-elles pas aussi le produit d'un dopage institutionnel et donc d'une concurrence déloyale avec les territoires moins peuplés ?

\section{Da course au statut de métropole...}

L'histoire du statut administratif de métropole commence en France avec une première loi en 2010. Puis les pouvoirs publics, convaincus que l'avenir de la France passe de façon prioritaire par les agglomérations les plus peuplées, font voter en 2014 une loi « d'affirmation des métropoles $»^{1}$ qui abaisse le seuil démographique, à partir duquel il est possible de bénéficier de ce statut, de 500000 à 400000 habitants. Il en résulte quinze territoires disposant d'un statut réglementaire de métropole et des avantages financiers liés. Constatant que cette réglementation est financièrement avantageuse, à commencer par l'octroi de 10 millions d'euros par l'État à la création de toute nouvelle métropole ${ }^{2}$, des agglomérations moins peuplées font pression pour obtenir à leur tour ce statut. Cela débouche, en 2017, sur une loi d'où résulte la création de sept nouvelles métropoles, portant leur total à 22 .

Certes, la corrélation entre la taille d'une commune ou d'une agglomération et son attractivité est loin d'être parfaite ${ }^{3}$. Pour la période 1975-2011, le résultat des métropoles françaises est très contrasté et parfois décevant ${ }^{4}$ en dépit des décisions de concentration d'un certain nombre de services publics 5 .

1. Soit douze métropoles de droit commun : Nice, créée dès la loi du 16 décembre 2010 ; Bordeaux, Brest, Grenoble, Lille, Montpellier, Nantes, Rennes, Rouen, Strasbourg, Toulouse, puis Nancy et trois métropoles à statut particulier (Paris, Lyon et Aix-Marseille) via la loi du 27 janvier 2014 de « modernisation de l'action publique territoriale et d'affirmation des métropoles », dite « loi MAPTAM »; et sept nouvelles métropoles résultant de la loi du 28 février 2017 : Clermont-Ferrand, Dijon, Metz, Orléans, SaintÉtienne, Toulon et Tours.

2. Ville, Frédéric, Réforme territoriale. Retour à la case démocratie!, Nantes, Salientes Éditions, 2019

3. Cf. Dumont, Gérard-François, « Les ressorts du développement local. La dynamique des territoires en France », Futuribles, $n^{\circ}$ 427, novembre-décembre 2018 ; Lugan, Jean-Claude, «Une petite ville en zone rurale peut-elle se développer? L'exemple de Figeac dans le sud-ouest de la France », Population \& Avenir, $n^{\circ} 743$, mai-juin 2019 ; Dumont, Gérardsud-ouest de la France », Population \& Avenir, n 743, mai-juin 2019 ; Dumont, Gérard-

4. Poupard, Gilles, « Développement local et emploi productif : un monopole des métropoles? », Population \& Avenir, n 725, novembre-décembre 2015.

5. Notamment sous l'effet de la révision générale des politiques publiques (RGPP) engagée en 2007 ; puis, fin 2012, de la « modernisation de l'action publique » (MAP).
Mais, avec les réglementations décidées au cours des années 2010, la donne a changé. Elle pourrait se traduire par des créations d'emplois significatives dans les métropoles. En effet, ces dernières reçoivent par habitant des dotations financières de l'État pouvant être jusquà trois fois plus élevées que pour les collectivités territoriales les moins peuplées. Il y a une prime à la taille dont les métropoles sont les plus grands bénéficiaires. Celui qui a plus peut donc faire davantage et, d'ailleurs, le nombre de fonctionnaires territoriaux pour mille habitants est quasiment corrélé avec la taille démographique des collectivités territoriales ${ }^{6}$.

\section{D...engendre automatiquement emplois directs et emplois induits}

Plus généralement, les territoires à statut de métropole, disposant d'une manne financière supplémentaire de la part de l'État, peuvent en conséquence renforcer leur attractivité. En outre, parmi les 22 métropoles, celles devenues capitales d'une région fusionnée depuis le $1^{\mathrm{er}}$ janvier 2016, soit Lille, Strasbourg, Rouen, Dijon, Lyon, Bordeaux et Toulouse, ont une situation avantagée. Il n'est pas rare que des fonctions auparavant existantes dans les capitales des ex-régions aient été transférées dans la capitale de la région fusionnée. Nombre d'organisations professionnelles, syndicales, associatives ont dû également fusionner et installer leur siège régional unique près du pouvoir régional. Cela a pu aussi être le cas d'entreprises privées réorganisant leurs directions régionales. Et il faut considérer tous les emplois induits par ce qui précède, qu'il s'agisse de l'hôtellerie-restauration de la capitale de la région fusionnée, dont la demande s'est accrue, et des autres emplois induits, ceux nécessaires pour satisfaire la consommation (alimentation, habillement, logement, services, etc.) des nouveaux personnels et de leur famille.

selon qu'elles

résultent des

Un autre aspect de l'emploi dans les capitales de régions fusionnées tient au niveau des rémunérations. Les éléments aux meilleures rémunérations souhaitent exercer leurs activités professionnelles là où se trouve leur centre régional de décisions. Une partie des nouveaux emplois des capitales des régions fusionnées sont donc des personnes de catégories supérieures. Et ces personnes engendrent davantage d'emplois induits, puisqu'il est démontré que le nombre d'emplois induits pour cent cadres est nettement supérieur à celui engendré par cent personnes des professions intermédiaires et plus encore par cent employés.

En conséquence, il importerait que l'étude des évolutions de l'emploi dans les métropoles conduise à distinguer leur double nature soit, d'une part, les hausses d'emploi liées au statut de ces territoires, résultant donc d'une sorte de dopage de ces territoires par les décisions réglementaires et financières de l'État et, d'autre part, les emplois dus à une véritable nouvelle attractivité.

6. Pour les communes, le taux d'administration est de 18,4 personnes employées (précisément d'EQTP : équivalent temps plein) pour 1000 habitants dans les communes de 300000 habitants ou plus contre 10,8 dans les communes de 2000 à 3500 habitants ; cf. Les collectivités locales en chiffres 2019, DGCL, juin 2019. 